\title{
Die Etablierung klientenbezogener Monitoringsysteme in der ambulanten Suchtkrankenhilfe am Beispiel der Hamburger Basisdatendokumentation (BADO)*
}

\section{Peter Lindlahr}

Freie und Hansestadt Hamburg, Amt für Gesundheit, Behörde für Arbeit, Gesundheit und Soziales, geschäftsführender Vorstand BADO e.V.

\begin{abstract}
Zusammenfassung: Mit zunehmender Etablierung der Hamburger Basisdatendokumentation (BADO) ist es gelungen, in den ambulanten Suchthilfeeinrichtungen ein einheitliches Dokumentationssystem zu realisieren, das hinsichtlich Erhebungsumfang, Datenindividualisierbarkeit und mittlerweile dokumentierter Fallzahl das umfassendste regionale Monitoringsystem der Bundesrepublik darstellt. Im Erhebungsjahr 2000 konnten zu 8960 Personen, die ambulante Beratungsoder Behandlungseinrichtungen für Alkohol- und Drogenabhängige aufgesucht haben, insgesamt 10777 Datensätze erhoben werden. Dies sind nicht nur 2011 Personen (29\%) mehr als im Vorjahr und damit ein deutlicher Zuwachs an Datenquantität, auch die Vollständigkeit der Angaben (Datenqualität) nimmt kontinuierlich zu. Die BADO als differenziertes Instrument zielgruppengerichteter Gesundheitsberichterstattung ermöglicht ein Monitoring gleichermaßen zu Lebensverhältnissen und Konsumtrends wie auch zum Inanspruchnahmeverhalten bezüglich der Hilfeangebote bei Alkohol- und Drogenabhängigen in der Metropolregion Hamburg. Hieraus abgeleitete Synergieeffekte, Koordinierungsnotwendigkeiten und strukturelle Weiterentwicklungen von Hilfesystemen eröffnen Praxis und Politik auf evidenz-basierter Grundlage umfassende Steuerungspotenziale. Sie zu nutzen, wird nicht nur regionale Aufgabe sein, sondern zugleich auch Herausforderung für bundes- und EUweite Netzwerke.
\end{abstract}

Schlüsselwörter: Dokumentationssyteme - Alkoholabhängigkeit - Konsumtrends - geschlechtsspezifische Suchtfaktoren - Heroinabhängigkeit - Soziale Desintegration Reichweite zielgruppenspezifischer Hilfen

The Documentation System "Hamburger Basisdatendokumentation" (BADO): Having succeeded to establish a monitoring system for drug and alcohol addicts in outpatient treatment at the level of a local treatment system, Hamburg's BADO, is meanwhile - regarding to sample sizes, data recognition and number of included cases - the largest regional data-based monitoring system in Germany. In 2000, the number of individual cases in the data file has increased by $2,011(29 \%)$ up to 8,960 persons, who contacted outpatient counselling and treatment services for alcohol- and drug dependants. As data quantity and data quality have

Suchttherapie 2001; 2: 231-234

(c) Georg Thieme Verlag Stuttgart · New York ISSN 1439-9903 been improved, a wide-ranged instrument has been created to enable social services as well as politics in starting evidence-based networking programmes.

Key Words: Documentation System - Alcohol Dependence - Consuming Trends - Gender and Addiction - Heroin Addicts - Social Desintegration - Range of Services

\section{Methodische Grundlagen [1]}

In mittlerweile 37 ambulanten Hamburger Suchthilfeeinrichtungen werden die im Rahmen des Beratungs- und Betreuungsgeschehens erhobenen klientenbezogenen Angaben in ein standardisiertes Erhebungsmanual übernommen und einer zentralen Auswertungsstelle, dem Institut für Sozialpädagogik und Sozialarbeit (ISS.V.) in Frankfurt am Main, zur Verfügung gestellt [1-4]. Das Spektrum der Angebotsprofile reicht von niedrigschwelligen Kontakt- und herkömmlichen Drogenberatungsstellen (24\%), Alkoholberatungsstellen und ambulanten Alkohol-Therapieeinrichtungen (16\%), suchtmittelübergreifenden Einrichtungen (16\%), Substitutionseinrichtungen (Methadonambulanzen) sowie Einrichtungen der psychosozialen Betreuung Substituierter (30\%) bis hin zur aufsuchenden Beratung im Strafvollzug (11\%).

Durch die in den Betreuungsprozess integrierte und daher weitgehend akzeptierte Form der „begleitenden Erhebung“ bedarf es keines gesonderten Fragebogens und auch keiner obligatorisch zu erhebenden Mindestmenge im Sinne eines Kerndatensatzes. An dessen Stelle tritt ein Manual, in dem nur erhoben wird, was im individuellen Beratungs- oder Betreuungszusammenhang ohnehin thematisiert worden ist. Die erhobenen Befunde weisen damit eine unmittelbare funktionale Zuordnung zum jeweiligen Betreuungsgeschehen auf. Im Jahr 2000 flossen auf diese Weise 10777 Datensätze von 8960 individualisierbaren Personen (Steigerung gegenüber dem Vorjahr: 29\%, Personen mit mehreren Datensätzen: 13\%) in die Erhebung ein. Anhand der Angaben zum Konsum psychoaktiver Substanzen konnten 8451 Klientinnen und

\footnotetext{
* BADO e.V. ist ein 1996 in Hamburg gegründetes Joint-Venture-Projekt von freien Trägern der Suchtkrankenhilfe und der Landesgesundheitsbehörde, das allein der Förderung und Koordinierung der gemeinsam entwickelten Basisdatendokumentation dient. Die Ausführungen des Verfassers stellen keine dienstliche Äußerung dar.
} 
Klienten (94,3\%) den beiden Hauptsubstanzgruppen „Alkohol“ und „illegale Drogen“ zugeordnet werden.

Die Individualisierbarkeit der Daten wird durch ein zweistufiges Datenschutzkonzept gewahrt: unter Beachtung datenschutzrechtlicher Vorgaben werden von drei Elementen (Personalcode, Items, Registriernummer) nach der Erhebung in der für die Auswertung anonym bleibenden Einrichtung nur noch jeweils zwei zusammengeführt, nämlich Personalcode und Registriernummer in einer externen Datenbank sowie Registriernummer und inhaltliche Angaben zu den Items beim Auswertungsinstitut. Hierdurch werden sowohl die Erkennung von Mehrfachaufnahmen innerhalb eines Jahres als auch von Weiterbetreuungen oder Wiederaufnahmen im Verlauf der Folgejahre auf einrichtungsübergreifender Ebene möglich. So liegen von 1389 Klientinnen und Klienten des Jahres 2000 korrespondierende Datensätze aus zwei Jahren, von 887 Personen Daten aus drei Jahren und von 562 Personen sogar aus vier Jahren vor. Der infolge des prozessbegleitenden Charakters der BADO systemimmanente Anteil von Missing Data erfordert permanente Bemühungen zur weiteren Optimierung der Datenqualität. Ebenso wie die differenzierte Datenauswertung erfolgen auch Missing-Analysen substanz- und geschlechtsbezogen. So hat sich die Datenqualität in den Bereichen Beziehung, Ausbildung, Einkommen/Schulden und bei den Behandlungsdaten erheblich verbessert. Durch gezielte Analysen unter Berücksichtigung der datenerhebenden Einrichtungsart und der Betreuungsdauer wird in den Folgejahren das Vollständigkeitsniveau nochmals deutlich angehoben werden können.

Im Jahr 2000 verdoppelte sich die Anzahl der datenerhebenden Einrichtungen mit alkoholabhängigen Klienten von sechs auf zwölf, wenngleich die Erreichungsquote bei geschätzten bis zu 65000 Alkoholabhängigen in Hamburg immer noch bei unter $10 \%$ liegt. Die BADO vermag damit operativ lediglich die von öffentlich finanzierten Einrichtungen erreichte Teilpopulation abzubilden, nicht jedoch den beträchtlichen Teil der Alkoholselbsthilfe. Gleichwohl haben die intensiven Bemühungen um eine stärkere strukturelle Integration des Alkoholsektors in die Gesamtdokumentation inzwischen Früchte getragen, da hier immer stärker auch Vergleiche auf Systemebene möglich werden. Hingegen bildet die BADO bereits heute den ganz überwiegenden Klientenanteil der ambulanten Drogenhilfeeinrichtungen Hamburgs ab: mittlerweile 31 an BADO beteiligte ambulante Einrichtungen für Konsumenten illegaler Drogen stellen bei insgesamt 35 derartigen Einrichtungen in Hamburg einen Anteil von 86 \% dar. Unter Berücksichtigung divergierender Schätzungen zur Gesamtprävalenz der intravenösen Drogenabhängigkeit in Ham-

Tab.1

\begin{tabular}{lrrr}
\hline & $\mathrm{n}=$ & Prozent & Gültige Prozente \\
Alkohol & 3193 & $35,6 \%$ & $37,8 \%$ \\
illegale Drogen & 5258 & $58,7 \%$ & $62,2 \%$ \\
gültig & 8451 & $94,3 \%$ & $100,0 \%$ \\
keine substanzbezo- & 207 & $2,3 \%$ & \\
$\begin{array}{l}\text { gene Differenzierung } \\
\text { fehlende Angaben }\end{array}$ & 302 & $3,4 \%$ & \\
gesamt & 8960 & $100,0 \%$ & \\
& & &
\end{tabular}

burg, die von 7000 bis 12000 reichen, weist BADO eine Erreichungsquote zwischen 43,8 und 79,4\% auf. Retrospektiv lassen sich auf der Basis des Ausgangsjahres 1997 unter Addition der in den Jahresverläufen der BADO neu hinzugekommenen, aber bereits langjährig abhängigen Klienten statistische Annäherungen zur Schätzung der Gesamtprävalenz durchführen. Auf der Datenbasis der Jahre 1997 bis 2000 ergibt sich für das Jahr 1997 mit diesem methodischen Ansatz eine retrospektive Schätzzahl von rund 11200 Drogenabhängigen.

\section{Aktuelle Befunde}

Von den im Jahr 2000 dokumentierten 3193 Personen mit Alkoholproblemen sind 65\% Männer und 35\% Frauen, davon insgesamt 5\% ausländischer Nationalität. Im Durchschnitt sind die Männer und Frauen mit Alkoholproblemen ca. 44 Jahre alt und bereits seit 13 Jahren (Frauen) bzw. 14 Jahren (Männer) abhängig (Tab. 1).

5234 Personen im Bereich illegaler Drogen sind in der Basisdokumentation erfasst, davon $72 \%$ Männer und $28 \%$ Frauen. Der Anteil deutscher Drogenabhängiger liegt bei 85\% (Männer) bzw. 92\% (Frauen). Das Durchschnittsalter beträgt bei drogenabhängigen Männern 34 Jahre und bei Frauen 31 Jahre. Hierbei ist seit Beginn der BADO im Jahr 1997 [4] ein ständiger und langsamer Anstieg des Durchschnittsalters zu verzeichnen, obgleich dies keine Abnahme des relativen Anteils junger Männer und Frauen bewirkt. Das Alter zu Beginn des Alkoholkonsums liegt sowohl bei Männern als auch bei Frauen zwischen 14 und 15 Jahren, bei Cannabiskonsum liegt dieses Alter bei 16 Jahren. Der Konsumbeginn bei Heroin und Kokain liegt hingegen im Alter zwischen 20 und 23 Jahren. Das Durchschnittsalter beim subjektiv erlebten Beginn der Abhängigkeit liegt bei den Männern zwischen 20 und 21 Jahren und bei den Frauen zwischen 19 und 20 Jahren.

Wie im Jahr 1999 [2] weisen Frauen ein deutlich niedrigeres Einstiegsalter beim Konsum von Heroin, Kokain und psychotropen Medikamenten auf als Männer. Gleiches gilt für den Beginn der Substitutionsbehandlung.

Die durchschnittliche Beratungs- oder Betreuungsdauer differiert mit der Art der Einrichtung. Hierbei fällt auf, dass drogenabhängige Frauen länger als drogenabhängige Männer die einrichtungsspezifischen Angebote wahrnehmen. Hinsichtlich der Verweildauern in den Einrichtungen weisen die in BADO dokumentierten klientenbezogenen Verläufe einen deutlichen Schwerpunkt in einem aktuellen Zeitraum von weniger als zwei Jahren auf (siehe Tab. 2)

Die Mehrheit der Personen mit Alkoholproblemen konsumierte ausschließlich Alkohol, 15\% konsumierten neben Alkohol noch weitere psychotrope Substanzen, vorwiegend Sedativa und Schmerzmittel (Frauen) sowie Cannabis (Männer). Unter den Personen in der Substanzgruppe illegaler Drogen dominiert bei $85 \%$ der Gebrauch von Opioiden. 74\% der Männer und 70\% der Frauen, die in den letzten 30 Tagen Heroin konsumierten, applizierten Heroin intravenös. 


\begin{tabular}{|c|c|c|c|c|}
\hline $\begin{array}{l}\text { Verweildauern } \\
\text { Betreuungsbeginn ... }\end{array}$ & $\begin{array}{l}\text { Frauen Alkohol } \\
{[n=1077]}\end{array}$ & $\begin{array}{l}\text { Männer Alkohol } \\
{[n=1958]}\end{array}$ & $\begin{array}{l}\text { Frauen illegale } \\
\text { Drogen [n=1419] }\end{array}$ & $\begin{array}{l}\text { Männer illegale } \\
\text { Drogen [n=3702] }\end{array}$ \\
\hline ...im laufenden Jahr & $57.9 \%$ & $59,2 \%$ & $61,0 \%$ & $66,0 \%$ \\
\hline ...im Vorjahr & $26,6 \%$ & $27,7 \%$ & $21,4 \%$ & $19,4 \%$ \\
\hline ...vor zwei Jahren & $7,6 \%$ & $8,9 \%$ & $6,9 \%$ & $5,9 \%$ \\
\hline ...vor mehr als drei Jahren & $2,5 \%$ & $1,7 \%$ & $7,1 \%$ & $5,6 \%$ \\
\hline durchschnittl. Dauer & 11 Monate & 10 Monate & 14 Monate & 12 Monate \\
\hline
\end{tabular}

Tab. 2

48\% der Männer und 46\% der Frauen konsumieren Kokain und/oder Crack. Auch Kokain wird von der Mehrheit der Konsumentinnen (59\%) und Konsumenten (65\%) intravenös konsumiert. Im Vergleich zum Vorjahr zeigt sich ein Anstieg des Crack-Konsums von $1,6 \%$ auf $4,4 \%$ bei den männlichen und von 2,4\% auf 6,4\% bei den weiblichen Drogenabhängigen. Der Anteil derjenigen, die angeben, schon einmal Crack konsumiert $\mathrm{zu}$ haben, ist damit auf weiterhin niedrigem Niveau, jedoch seit dem Jahr 1999 deutlich angestiegen von insgesamt 99 auf 392 (71\% Männer und 29\% Frauen). Eine differenzierte Auswertung der Daten dieser Gruppe zeigt, dass vor allem Männer und Frauen der Altersklassen 22 bis 29 Jahre und 30 bis 39 Jahre Erfahrungen mit Crack haben. Cannabiskonsum (36\% Männer, 31\% Frauen), Alkoholkonsum (32\% Männer, 29\% Frauen) und Sedativa, Benzodiazepine, Barbiturate (14\% Männer, 16\% Frauen) werden bei Drogenabhängigen im Beratungs- und Betreuungsprozess ebenfalls thematisiert und fließen damit in die BADO ein. Die Gesamtheit von 8451 substanzspezifisch differenzierten Fällen aus Alkohol- und Drogenhilfeeinrichtungen weist folgende Verteilung zum Merkmal „Hauptdroge“ auf (siehe Tab. 3)

Insgesamt ist bei $80 \%$ der Personen mit Alkoholproblemen eine entsprechende Behandlungserfahrung (mindestens eine stationäre oder ambulante Entgiftung oder Entwöhnung) dokumentiert. Dabei weist die Teilpopulation aus den Alkoholberatungs- und Behandlungsstellen durchweg eine höhere Behandlungserfahrung als die in anderen Einrichtungen auf.

Bemerkenswert ist, dass fast die Hälfte der KlientInnen, die über zwei Jahre in den Alkoholberatungsstellen dokumentiert sind, in diesem Zeitraum abstinent wird oder bleibt.

Tab. 3

\begin{tabular}{lrrr} 
& Männer & Frauen & gesamt \\
& & & \\
\hline & & & \\
Alkohol & $51,8 \%$ & $49,9 \%$ & $51,1 \%$ \\
Opioide & $53,8 \%$ & $47,0 \%$ & $50,8 \%$ \\
Kokain und Crack & $31,1 \%$ & $27,8 \%$ & $29,0 \%$ \\
Cannabis & $23,9 \%$ & $17,9 \%$ & $21,7 \%$ \\
Sedativa/Hypnotika & $9,3 \%$ & $10,3 \%$ & $9,5 \%$ \\
sonstige psychotrope & $3,6 \%$ & $4,4 \%$ & $3,8 \%$ \\
Substanzen & & & \\
Stimulanzien & $0,9 \%$ & $1,0 \%$ & $0,9 \%$ \\
Schnüffelstoffe & $0,3 \%$ & $0,2 \%$ & $0,3 \%$ \\
n & 5.750 & 2.525 & $8.451 *$ \\
$*$ einschließlich Fälle ohne Angabe zum Geschlecht & \\
& &
\end{tabular}

Wegen einer körperlichen Erkrankung war in den letzten zwölf Monaten lediglich ein Fünftel (23\% Männer und 20\% Frauen) in stationärer klinischer Behandlung. Immerhin bei 36\% der Männer und 34\% der Frauen mit Alkoholproblemen sind akute gesundheitliche Beeinträchtigungen dokumentiert, wobei Männer ab 44 Jahren häufiger gesundheitliche Probleme aufweisen, die sie akut beeinträchtigen. Jüngere Frauen bis 43 Jahre waren hingegen häufiger in ambulanter und/oder stationärer psychotherapeutischer Behandlung.

69\% der opiatabhängigen Männer und 71\% der Frauen befinden sich in einer Substitutionsbehandlung. 78\% der Drogenkonsumenten und $81 \%$ der -konsumentinnen verfügen über Behandlungserfahrungen in ambulanter oder stationärer Entgiftung und Entwöhnung, in den letzten zwölf Monaten wurden 24\% der Frauen und 19\% der Männer im Krankenhaus wegen körperlicher Probleme behandelt. Unter den Erkrankungen dominiert Hepatitis C mit $41 \%$ (ca. 50\% der Substituierten) Die HIV-Prävalenz bei den Klientinnen und Klienten insgesamt beträgt rund $5 \%$, bei den Substituierten ist sie mit $6 \%$ nur leicht erhöht. $17 \%$ der drogenkonsumierenden Männer und 24,3\% der Frauen wurden zusätzlich psychotherapeutisch oder psychiatrisch ambulant oder stationär behandelt, hierunter mehr Substituierte als andere Personen.

Von den Alkoholpatienten haben lediglich 3\% keinen Schulabschluss und 20\% keine abgeschlossene Berufsausbildung, etwa ein Drittel ist erwerbslos. 13\% der Drogenabhängigen haben keinen Schulabschluss, 2\% haben einen Sonderschulabschluss. Das Qualifikationsniveau der Schulabschlüsse ist insgesamt niedrig. 50\% der Männer und 40\% der Frauen haben eine abgeschlossene Berufsausbildung, ein Ergebnis, das seit 1997 fast konstant ist [4]. 92\% der alkoholabhängigen Männer und $97 \%$ der Frauen verfügen über eigenen Wohnraum, wobei jedoch der Anteil alkoholabhängiger Männer ohne eigenen Wohnraum faktisch höher liegen dürfte, da BADO keine Daten aus speziellen Einrichtungen zur Betreuung von Obdachlosen aufweist. 40\% der alkoholabhängigen Männer und 28\% der Frauen leben allein. 46\% der Männer haben Kinder, mit denen 34\% (allein oder mit Partnerin) zusammenleben. Demgegenüber haben 59\% der alkoholabhängigen Frauen Kinder, mit denen 46\% zusammenleben. Die in den Einrichtungen betreuten Frauen und Männer haben insgesamt ca. 1700 Kinder. 71\% drogenabhängiger Männer und $79 \%$ der Frauen haben einen eigenen Wohnraum; über den Erhebungszeitraum von 4 Jahren sind die Angaben über akute Wohnungslosigkeit leicht rückläufig. Insbesondere der Anteil derjenigen, bei denen dokumentiert wird, dass sie „auf der Straße“ leben, ist kontinuierlich gesunken. 
$43 \%$ der drogenabhängigen Väter und 22\% der Mütter leben allein. Die in den Drogenhilfeeinrichtungen betreuten Männer und Frauen haben insgesamt ca. 2.500 Kinder (33\% Männer, von denen $32 \%$ mit den Kindern zusammenleben, 44\% Frauen, von denen 53\% mit den Kindern zusammenleben). Von den Frauen ohne Kinder leben 51\% allein, 30\% mit Partnern und alle übrigen in anderen Kontexten. Männer und Frauen mit Kindern sind erheblich häufiger als diejenigen ohne Kinder an Einrichtungen der psychosozialen Betreuung für Substituierte angebunden.

Wegen der insgesamt verbesserten Datenqualität konnte die Variable „Soziale Desintegration“ erstmals auch für die Substanzgruppe Alkohol berechnet werden. Hierbei werden von den Variablen zur sozialen Situation drei für diese Dimension relevante Items ausgewählt, für die ausreichend Daten vorlagen: Partnerbeziehungen, Wohnsituation und Erwerbsstatus. Diese Variablen wurden mit dem Ziel dichotomisiert, die Vielzahl der jeweiligen Kategorien auf zwei zu reduzieren, von denen dann eine als Indikator für soziale Desintegrationserscheinungen gewertet werden kann. Hierbei werden nur diejenigen Fälle ausgewählt, bei denen für diese drei Variablen gültige Angaben vorlagen. Nach Bildung von vier Clustern, die eine soziale Desintegration andeutende Merkmalsausprägung gestuft abbilden, lässt sich bei $28 \%$ der Alkoholabhängigen eine starke und bei $72 \%$ eine geringe soziale Desintegration nachweisen. Inwieweit sich diese Verteilung als „angebotsinduziert“ darstellt, da die betreffende Klientengruppe eher von höherschwelligen Einrichtungen beraten und behandelt wird, kann erst dann abschließend beurteilt werden, wenn sich noch stärker als bisher niedrigschwellige Einrichtungen an BADO beteiligen und sich dann ein veränderter Anteil bei stark sozial Desintegrierten entwickelt. Alkoholabhängige Männer (31\%) sind häufiger als Frauen (23\%) stark sozial desintegriert. Wie auch bei den Drogenkonsumenten besteht ein enger Zusammenhang zwischen Hafterfahrung und dem Ausmaß der sozialen Desintegration (54\%) bei den Männern mit Alkoholproblemen. Insgesamt sind 56\% der Drogenabhängigen (Männer 60\%, Frauen 47\%) als stark sozial desintegriert und $44 \%$ als gering sozial desintegriert zu klassifizieren. Die höchsten Anteile stark sozial desintegrierter Männer finden sich in den Einrichtungen der psychosozialen Betreuung für Substituierte. Stark sozial desintegrierte Frauen dagegen sind am häufigsten in Kontakt- und Drogenberatungsstellen dokumentiert.

\section{Diskussion der Ergebnisse}

An die Etablierung von Dokumentationssystemen in der ambulanten Suchthilfepraxis ist die grundlegende Erwartung zu stellen, jenseits unterschiedlichster suchtwissenschaftlicher Interessenlagen für die sozialarbeiterische und therapeutische Praxis ein Reflektions- und Steuerungsinstrument zu erlangen und zugleich für die staatliche Versorgungsplanung wesentliche Entwicklungsparameter aufzuzeigen. Die Hamburger BADO ist in der Lage, für eine Population von knapp 9000 Personen Lebensumstände, suchtspezifische Parameter und Inanspruchnahmeverhalten in einer Weise abzubilden, die es - bei ständig weiter steigender Datenqualität und Datenquantität - erlaubt, neue Maßstäbe im Sinne einer evidenzbasierten Suchtkrankenhilfe zu setzen.
Hierbei wird es in der Weiterentwicklung dieses Instruments auf der Auswertungsebene darum gehen, die zunehmende Datenfülle methodisch stärker zu kanalisieren, Aussagen und Verknüpfungen noch stärker zu validieren und Plausibilitäten durch noch gezieltere Hinzuziehung von Missing-Analysen zu erhöhen. Die Beteiligten sind sich darin einig, die Basisdatendokumentation durchaus im Gesamtverständnis einer zeitgemäßen und an den Anforderungen einer Metropolregion wie Hamburg ausgerichteten Gesundheitsberichterstattung $\mathrm{zu}$ begreifen, hierbei aber jederzeit der Suchthilfepraxis eine umfassende Möglichkeit zu gewähren, auf die Basisdatendokumentation als empirisch gesicherte Grundlage der eigenen Positionierungen zurückgreifen zu können.

Das aktuelle Berichtsjahr 2000 weist hierzu in zahlreichen Punkten Prozess- und Ergebnisqualitäten nach, etwa bei der Erreichung von Abstinenz bei den Alkoholpatienten. Latent vernachlässigte Handlungsbedarfe (beispielhaft hier: die hohe Kinderzahl sowohl im Alkohol- als auch im Drogenbereich oder die abermals bestätigte relativ höhere Attraktivität von Heroin für die Gruppe junger Frauen) werden ebenso in Erinnerung gerufen wie umgekehrt auch aktuell aufkommende politische Diskussionen [5] durch entsprechende Befunde empirisch unterlegt oder relativiert (hier: die Steigerung des Crack-Konsums bewegt sich weiterhin auf eher niedrigem Gesamtniveau) werden können. Somit eröffnet BADO weitergehende Perspektiven für die Projektsteuerung, für die bundes- und EU-weite Vernetzung regionaler Hilfesysteme und nicht zuletzt auch für hierauf bezogene staatliche Planungsentscheidungen.

\section{Literatur}

${ }^{1}$ Schmid M, Simmedinger R, Vogt I. Zur umfassenden Darstellung der hier referierten Dokumentations-Ergebnisse für das Jahr 2000: Ambulante Suchthilfe in Hamburg. Statusbericht 2000 zur Hamburger Basisdatendokumentation im ambulanten Suchthilfesystem. Frankfurt am Main

${ }^{2}$ Schmid M, Simmedinger R, Vogt I. Ambulante Suchthilfe in Hamburg. Statusbericht 1999 zur Hamburger Basisdatendokumentation im ambulanten Suchthilfesystem. Frankfurt am Main

${ }^{3}$ Schmid M, Simmedinger R, Vogt I. Ambulante Suchthilfe in Hamburg. Statusbericht 1998 zur Hamburger Basisdatendokumentation im ambulanten Suchthilfesystem. Frankfurt am Main

${ }^{4}$ Schmid M, Vogt I. Ambulante Suchthilfe in Hamburg. Statusbericht 1997 zur Hamburger Basisdatendokumentation im ambulanten Suchthilfesystem. Frankfurt am Main

${ }^{5}$ Crack-Studie: in den Metropolen wächst die Sucht nach Steinen. Ärzte-Zeitung 3.9.01; 155

\section{BADO e.V.}

c/o Therapiehilfe e. V. Hasselbrookstraße 94 a 22089 Hamburg 